\title{
Synthesis and Antimicrobial Activity of Naphthylamine Analogs Having Azetidinone and Thiazolidinone Moiety
}

\author{
Bhawna Chopra ${ }^{1,2 *}$, Ashwani K. Dhingra ${ }^{1}$, Ram P. Kapoor ${ }^{1}$ and Deo N. Parsad ${ }^{3}$ \\ ${ }^{1}$ Guru Gobind Singh College of Pharmacy, Yamuna Nagar-135001 Haryana, India; \\ ${ }^{2}$ Research Scholar, IK Gujral Punjab Technical University, Jalandhar-144001, Punjab, India; \\ ${ }^{3}$ Shivalik College of Pharmacy, Nangal-140126, Punjab, India
}

\begin{abstract}
Background and Objective: To synthesize 2-naphthylamine analogs containing azetidin-2-one (4a-g) and thiazolidin-4-one (5a-g) ring moiety, with the aim of finding new potent antimicrobial agents.
\end{abstract}

Methods: The antimicrobial activities (antibacterial and antifungal) of the newly-prepared compounds were tested in vitro against bacterial cultures (Bacillus subtilis, Staphylococcus aureus, Escherichia coli, and Pseudomonas aeruginosa) and fungal culture (Candida albicans) using agar plate diffusion antimicrobial bioassay. The structures of the title compounds were supported by their spectral data (IR, ${ }^{1} \mathrm{H}$ NMR and ${ }^{13} \mathrm{C} N M R$ ).

Results: The synthetic methodology used for the synthesis of the title compounds is shown in Scheme 1 in the paper. Among all the prepared analogs, four compounds (4a, 4e, 4g and 4f) exhibited broad spectrum activity, as compared to the standard drug (ampicillin). Another three compounds (3b, 5b and 5e) showed remarkable antifungal activity, as compared with the standard drug (amphotericin B).

Conclusions: The present investigation led to the synthesis and biological evaluation of naphthylamine analogs having azetidin-2-one and thiazolidin-4-one heterocyclic nucleus/moiety.

\section{Introduction}

The discovery, development and identification of biologically active antimicrobial compounds have gained a lot of importance in recent years, even though there are a considerable number of adverse effects. Medicinal chemists have always been tried to design drug molecules that possess maximum therapeutic application while having minimum toxicity profile. Moreover, because of excessive use of antibacterial antibiotics, immunosuppressants and cytotoxins, opportunistic mycosis has become prominent. To combat the

Keywords: Antimicrobial; 2-Azetidinone; 4-Thiazolidinone; Schiff bases; Naphthalene.

Abbreviations: FTIR, Fourier-transform infrared spectroscopy; IR, infrared; Natom, number of atoms other than hydrogen; NMR, nuclear magnetic resonance; nOHNH, number of $\mathrm{H}-$ bond acceptor; nON, number of hydrogen bond donor; TMS, tetramethylasilane; TPSA, topological polar surface area; ZOI, zone of inhibition.

Received: February 22, 2017; Revised: August 01, 2017; Accepted: August 18, 2017 *Correspondence to: Bhawna Chopra, Guru Gobind Singh College of Pharmacy, Near Guru Nanak Khalsa College, Yamuna Nagar-135001, Haryana, India. Tel: +919896151755, Fax: 01732268381. E-mail: bhawna8486@gmail.com

How to cite this article: Chopra B, Dhingra AK, Kapoor RP, Parsad DN. Synthesis and Antimicrobial Activity of Naphthylamine Analogs Having Azetidinone and Thiazolidinone Moiety. J Explor Res Pharmacol 2017;2(4):105-112. doi: 10.14218/ JERP.2017.00005. increasing number of fungal pathogens and the growing burden of resistance, there is a need to develop new antimicrobial compounds.

Naphthalene analogs occupy a central place among medicinally important compounds, due to their diverse and interesting antibiotic properties. ${ }^{1}$ Napthalene is the simplest and the most important member of the arene class, in which two benzene rings are fused in ortho positions. The effectiveness of this structural feature is demonstrated by $\beta$-naphthol having almost the same carbon skeleton; it is commonly used as a dye, and reported to possess very good antimicrobial property. ${ }^{2}$ Several naphthalene nucleus-containing drugs are also available, such as nafacillin, naftifine, tolnaftate, terbinafine, etc. (Fig. 1), all of which play a vital role in the control of microbial infection. ${ }^{1}$

On the other hand, the imine and azomethine groups $(-\mathrm{N}=\mathrm{CH}-)$ have been found, respectively, in various natural, natural-derived and non-natural compounds (Fig. 2) and have been shown to be critical to their biological activities. ${ }^{3-5}$ They occupy a central place among medicinally important compounds due to their wide spectrum of biological properties, including antimicrobial, ${ }^{6}$ antiinflammatory, ${ }^{7}$ analgesic, ${ }^{8}$ anti-tubercular, ${ }^{8,9}$ antimycobacterial, ${ }^{10}$ antioxidant, ${ }^{11}$ antiviral, ${ }^{12}$ inhibitory, ${ }^{13}$ cytotoxic, ${ }^{14}$ anticonvulsant ${ }^{15}$ anti-proliferative, ${ }^{16}$ anticancer, ${ }^{17}$ and antifungal activities. ${ }^{18}$ Schiff bases of aromatic aldehydes with effective conjugation system are more stable than the aliphatic aldehydes. 


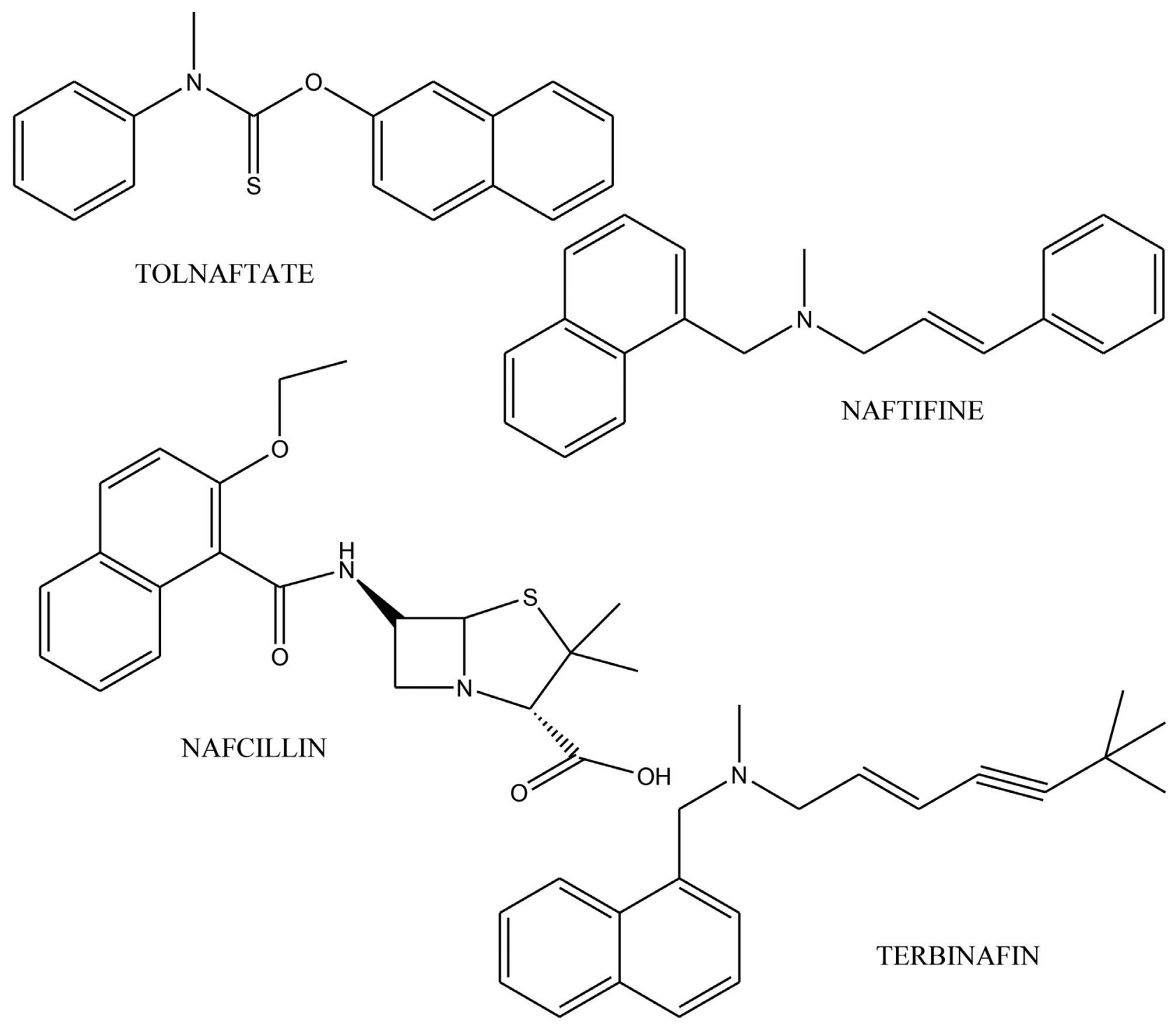

Fig. 1. Structure of clinically used naphthalene nucleus-containing antimicrobial drugs.

Moreover, numerous analogs of azetidinone andthiazolidinone moieties have also been well documented..$^{19-21}$ Various reports of studies of naphthalene and its based analogs bearing the azetidine and thiazolidone nucleus possess pharmacological potential. ${ }^{22,23}$ However, the biological activity of this class of compounds deserves further investigation. The purpose of this present study was to evaluate the antimicrobial potential of azetidinone and thiazolidinone analogs of napthylamine.

\section{Material and methods}

\section{Chemistry}

All the chemicals used were of synthetic grade and commercially procured from Qualigen (Mumbai, India) and CDH (New Delhi, India). Melting points were determined by open capillary tube method and are uncorrected. Fourier-transform infrared spectroscopy (FTIR) spectra (KBr pellet, neat) were recorded on a Perkin Elmer RX1 spectrophotometer and ${ }^{1} \mathrm{H}-$-nuclear magnetic resonance (NMR) and ${ }^{13} \mathrm{C}$ NMR spectra were recorded on a Brucker $300 \mathrm{MHz}$ spectrometer in $\left(\mathrm{CDCl}_{3}\right)$ using tetramethylsilane (TMS) as an internal reference and the chemical shifts are expressed in $\delta$ ppm. Progress of the reaction and purity of the compound was monitored by thin layer chromatography using a $0.2 \mathrm{~mm}$ thick aluminium sheet precoated with silica gel (60F 254; Merck, United States). Spots were visualized under UV $(254 \mathrm{~nm})$ and iodine chamber. The solvent was removed under reduced pressure, using a rotary evaporator (Buchi, United States). All other organic solvents used were of laboratory reagent grade, dried over anhydrous sodium sulfate and used as received.

\section{Synthesis of Schiff bases (3a-g)}

The appropriate aryl aldehydes $(0.01 \mathrm{~mol})$ were added to a solution of $\beta$-napthylamine $(1.43 \mathrm{~g}, 0.01 \mathrm{~mol})$ in ethanol $(20 \mathrm{~mL})$, followed by dropwise addition of concentrated sulphuric acid. The reaction 


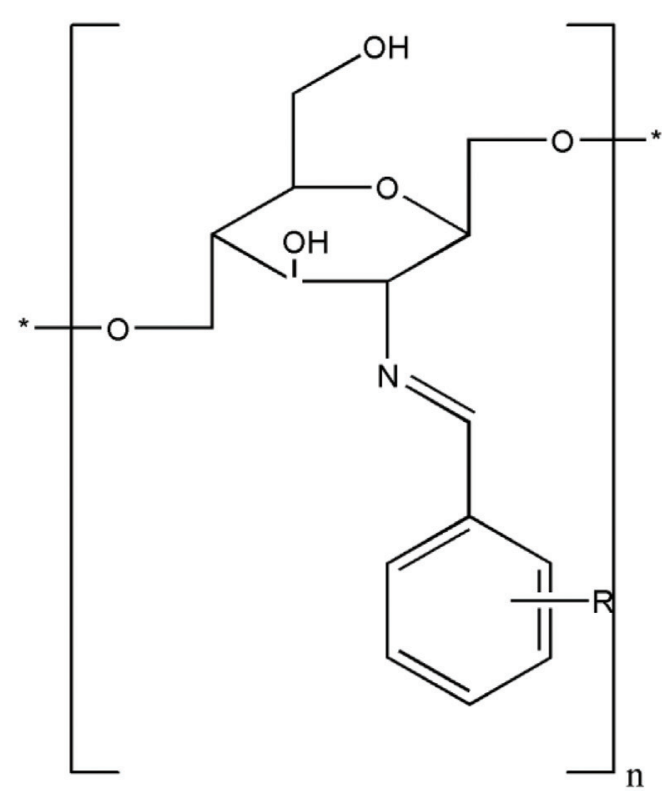

CHITOSAN DERIVED SCHIFF BASE (ANTIFUNGAL) NATURAL PRODUCT DERIVED<smiles>Oc1ccccc1/C=N/c1ccccc1O</smiles>

\section{N-(SALICYLIDENE)-2-HYDROXYANILINE (ANTIBACTERIAL) NON NATURAL COMPOUND}

Fig. 2. Structure of clinically used imine group-containing drugs.

mixture was then refluxed for $6-8 \mathrm{~h}$ and cooled. After cooling, the solid obtained was filtered, washed thoroughly with water, and dried. The pure product was crystallized from ethanol/water $(1 / 1 \mathrm{v} / \mathrm{v})$.

\section{Synthesis of 3-chloro-4-(substituted phenyl)-1-(naphthalen-6-yl) azetidin-2-one (4a-g)}

A solution of compound (3a-g, $0.01 \mathrm{~mol})$ in dioxane $(20 \mathrm{~mL})$ and triethylamine $(0.01 \mathrm{~mol})$ was mixed with continuous stirring. The total mixture was then cooled at $0-5{ }^{\circ} \mathrm{C}$ and a few drops of chloroacetyl chloride were added. After addition, the whole mixture was stirred for 5-6 h and then filtered. The filtrate was then refluxed for another 5-6 h and allowed to cool at room temperature. On cooling, a solid crude product separated out, which was filtered, dried and re-crystalized with ethanol.<smiles>NC(=O)N/N=C/c1ccc([N+](=O)[O-])o1</smiles>

NITROFYRAZONE NON NATURAL COMPOUND<smiles>COc1cc2cc(C)ncc2c(OC)c1-c1c(C)cc2cccc(OC)c2c1O</smiles>

\section{ANCISTROCLADIDINE (ANTIMALARIAL ) NATURAL PRODUCT}<smiles>CC(=O)Cc1ccc(C=NNC(N)=S)cc1</smiles>

THIOACETAZONE NON NATURAL COMPOUND

Synthesis of N-2-(substituted phenyl)-3-(naphthalen-6-yl) thiazolidin-4-one (5a-g)

A mixture of compound (3a-g, $0.01 \mathrm{~mol})$ and thioglycollic acid $(0.01 \mathrm{~mol})$ was refluxed on a heating mantle at $120-125^{\circ} \mathrm{C}$ for approximately $12 \mathrm{~h}$. The reaction mixture was cooled and treated with $10 \%$ sodium bicarbonate solution. The product was isolated and re-crystallized from methanol-dioxane (4:1). The overall reaction scheme followed is depicted in Scheme 1.

\section{4-(naphthalen-3-ylimino methyl) phenol 3a}

Molecular formula $\mathrm{C}_{17} \mathrm{H}_{13} \mathrm{NO}$; melting point $116-117{ }^{\circ} \mathrm{C}$; yield $60 \%$. Infrared (IR) $\left(\mathrm{KBr}, \mathrm{cm}^{-1}\right) 3,045.0(\mathrm{CH}), 1,440-1,514(-\mathrm{H}-$ $\mathrm{C}=\mathrm{N}) .{ }^{1} \mathrm{H}-\mathrm{NMR}\left(300 \mathrm{MHz}, \mathrm{CDCl}_{3}, \mathrm{TMS}=0\right) \delta: 8.2(\mathrm{~s}, 1 \mathrm{H}, \mathrm{CH})$, 
<smiles>Nc1ccc2ccccc2c1</smiles>

1<smiles>[R]c1ccc(C=O)cc1</smiles>

a<smiles>[R]c1ccc(C=Nc2ccc3ccccc3c2)cc1</smiles>

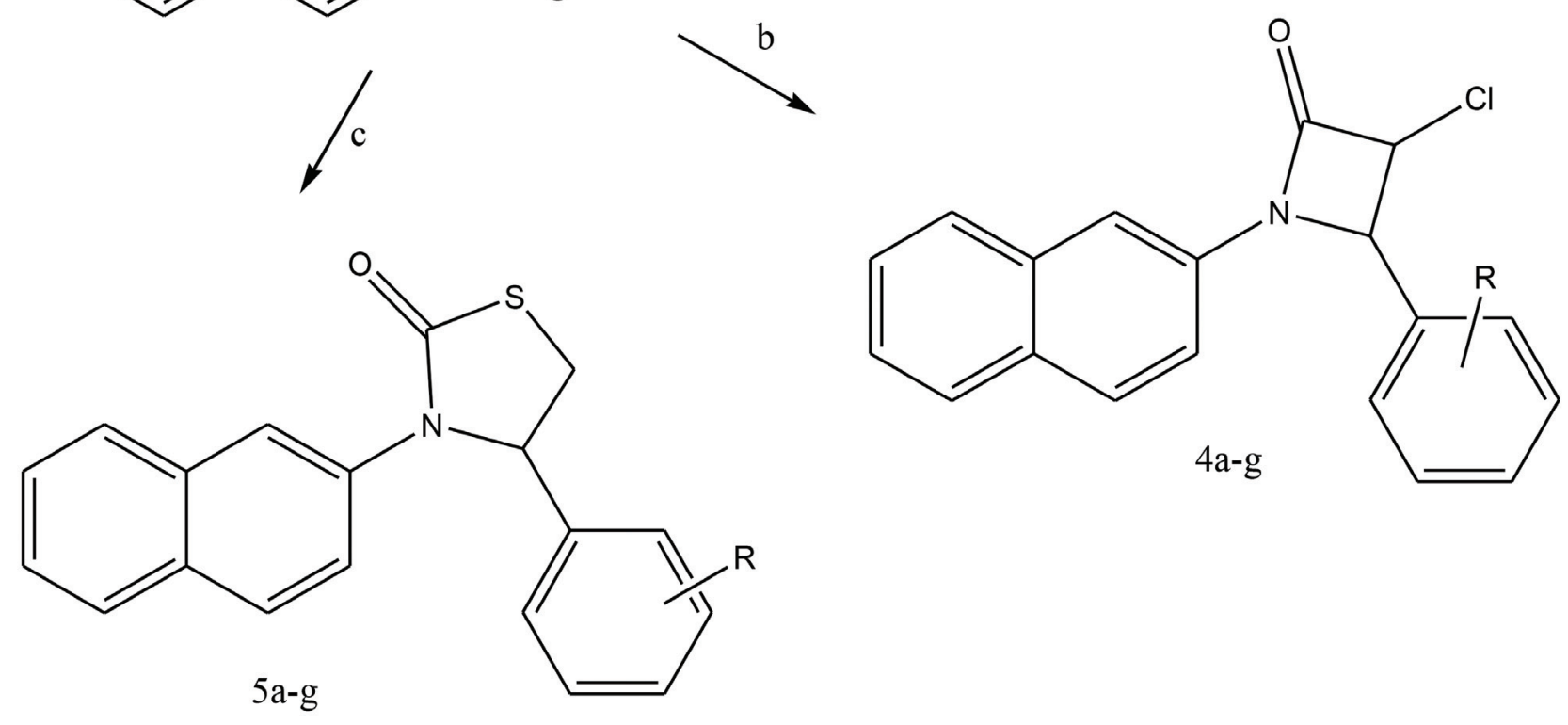

Scheme 1. Reagents and conditions. a. sulphuric acid, ethanol, 5-6 h reflux.; b. Triethylamine, dioxane, chloroacetyl chloride, stirring followed by 5-6 h reflux, ethanol.; c. Thioglycollic acid, $12 \mathrm{~h}$ reflux, $10 \%$ sodium bicarbonate solution.

$4.9(\mathrm{~s}, 1 \mathrm{H}, \mathrm{OH}), 6.7-7.4(\mathrm{~m}, 11 \mathrm{H}, \mathrm{Ar}-\mathrm{H}) .{ }^{13} \mathrm{C}-\mathrm{NMR}(300 \mathrm{MHz}$, $\left.\mathrm{CDCl}_{3}\right) \delta: 160.6,150.4,135.2,132.4,130,129.4,128.2,126.4$ $120.4,119.2,116$.

\section{3,4,5-trimethoxybenzylidenenaphthalen-2-amine 3b}

Molecular formula $\mathrm{C}_{20} \mathrm{H}_{19} \mathrm{NO}_{3}$; melting point $131-132{ }^{\circ} \mathrm{C}$; yield $60 \% .{ }^{1} \mathrm{H}-\mathrm{NMR}\left(300 \mathrm{MHz}, \mathrm{CDCl}_{3}, \mathrm{TMS}=0\right) \delta: 3.7\left(\mathrm{~s}, 9 \mathrm{H}, \mathrm{OCH}_{3}\right)$, $8.3(\mathrm{~s}, 1 \mathrm{H}, \mathrm{CH}), 6.6-7.4(\mathrm{~m}, 9 \mathrm{H}, \mathrm{Ar}-\mathrm{H}) .{ }^{13} \mathrm{C}-\mathrm{NMR}(300 \mathrm{MHz}$, $\left.\mathrm{CDCl}_{3}\right) \delta: 160.4,150.6,141.5,135,132.2,129.4,128.2,126.2$, 120.6, 119.2, 106.2, 56.2.

\section{3,4-dimethoxybenzylidene naphthalen-2-amine $3 \mathrm{c}$}

Molecular formula $\mathrm{C}_{19} \mathrm{H}_{17} \mathrm{NO}_{2}$; melting point $128-130{ }^{\circ} \mathrm{C}$; yield
$60 \% .{ }^{1} \mathrm{H}-\mathrm{NMR}\left(300 \mathrm{MHz}, \mathrm{CDCl}_{3}, \mathrm{TMS}=0\right) \delta: 3.6\left(\mathrm{~s}, 6 \mathrm{H}, \mathrm{OCH}_{3}\right)$, $8.1(\mathrm{~s}, 1 \mathrm{H}, \mathrm{CH}), 6.7-7.6(\mathrm{~m}, 10 \mathrm{H}, \mathrm{Ar}-\mathrm{H})$.

2-methoxy-4-(naphthalen-3-ylimino methyl) phenol 3d

Molecular formula $\mathrm{C}_{18} \mathrm{H}_{15} \mathrm{NO}_{2}$; melting point $114-115{ }^{\circ} \mathrm{C}$; yield $60 \% .{ }^{1} \mathrm{H}-\mathrm{NMR}\left(300 \mathrm{MHz}, \mathrm{CDCl}_{3}, \mathrm{TMS}=0\right) \delta: 3.7\left(\mathrm{~s}, 3 \mathrm{H}, \mathrm{OCH}_{3}\right)$, $5.0(\mathrm{~s}, 1 \mathrm{H}, \mathrm{OH}), 8.2(\mathrm{~s}, 1 \mathrm{H}, \mathrm{CH}), 6.7-7.4(\mathrm{~m}, 10 \mathrm{H}, \mathrm{Ar}-\mathrm{H}) .{ }^{13} \mathrm{C}-$ $\operatorname{NMR}\left(300 \mathrm{MHz}, \mathrm{CDCl}_{3}\right) \delta: 160.1,151.4,150.2,148,135.2,132.2$, $129.4,128,127.4,123,120.4,119.2,117,114.8,56$.

Benzylidenenaphthalen-2-amine 3e

Molecular formula $\mathrm{C}_{17} \mathrm{H}_{13} \mathrm{~N}$; Melting point $106-107{ }^{\circ} \mathrm{C}$; Yield $60 \%$; ${ }^{1} \mathrm{HNMR}\left(300 \mathrm{MHz}, \mathrm{CDCl}_{3}, \mathrm{TMS}=0\right) \delta: 8.1(\mathrm{~s}, 1 \mathrm{H}, \mathrm{CH})$, 
Chopra B. et al: Napthylamine analogs having heterocyclic moiety

7.2-7.6 (m, 12H, Ar-H).

3-nitrobenzylidenenaphthalen-2-amine $3 f$

Molecular formula $\mathrm{C}_{17} \mathrm{H}_{12} \mathrm{~N}_{2} \mathrm{O}_{2}$; melting point $122-123{ }^{\circ} \mathrm{C}$; yield $70 \% .{ }^{1} \mathrm{H}-\mathrm{NMR}\left(300 \mathrm{MHz}, \mathrm{CDCl}_{3}, \mathrm{TMS}=0\right) \delta: 8.5(\mathrm{~s}, 1 \mathrm{H}, \mathrm{Ar}-\mathrm{H})$, 8.3 (s, 1H, CH), 8.1-8.2 (d, 2H, Ar-H), 7.3-7.6 (m, 8H, Ar-H).

4-chlorobenzylidenenaphthalen-2-amine $3 \mathrm{~g}$

Molecular formula $\mathrm{C}_{17} \mathrm{H}_{12} \mathrm{C}_{1} \mathrm{~N}$; melting point $124-125{ }^{\circ} \mathrm{C}$; yield $65 \%$. ${ }^{1} \mathrm{H}-\mathrm{NMR}\left(300 \mathrm{MHz}, \mathrm{CDCl}_{3}, \mathrm{TMS}=0\right) \delta: 8.1(\mathrm{~s}, 1 \mathrm{H}, \mathrm{CH})$, 7.3-7.6 (m, 11H, Ar-H).

3-chloro-4-(4-hydroxyphenyl)-1-(naphthalen-3-yl)azetidin-2-one 4a

Molecular formula $\mathrm{C}_{19} \mathrm{H}_{14} \mathrm{ClNO}_{2}$; melting point $146-147^{\circ} \mathrm{C}$; yield $60 \%$; IR $\left(\mathrm{KBr}, \mathrm{cm}^{-1}\right) 3,450.5(\mathrm{NH}), 3,047.0(\mathrm{CH}), 1,754.1(\mathrm{C}=\mathrm{O})$, 1,624.9 (CO-NH), 1,448.7 (CN), 896.5, 750.5 and 677.8 $(\mathrm{C}=\mathrm{C})$, $679.8(\mathrm{C}-\mathrm{Cl}) .{ }^{1} \mathrm{H}-\mathrm{NMR}\left(300 \mathrm{MHz}, \mathrm{CDCl}_{3}, \mathrm{TMS}=0\right) \delta: 5.0(\mathrm{~s}, 1 \mathrm{H}$, $\mathrm{OH}), 5.2(\mathrm{~d}, 1 \mathrm{H}, \mathrm{CH}), 5.4(\mathrm{~d}, 1 \mathrm{H}, \mathrm{CH}), 6.8-7.4(\mathrm{~m}, 11 \mathrm{H}, \mathrm{Ar}-\mathrm{H})$. ${ }^{13} \mathrm{C}-\mathrm{NMR}\left(300 \mathrm{MHz}, \mathrm{CDCl}_{3}\right) \delta: 162.1,156.4,141.2,136.4,133.2$, $128,127.4,126.1,125.4,124,121.4,118.2,115,108.6,63,62.2$.

3-chloro-4-(3,4,5-trimethoxyphenyl)-1-(naphthalen-3-yl)azetidin2-one $4 b$

Molecular formula $\mathrm{C}_{22} \mathrm{H}_{20} \mathrm{ClNO}_{4}$; melting point $134-135^{\circ} \mathrm{C}$; yield $60 \%$; ${ }^{1} \mathrm{H}-\mathrm{NMR}\left(300 \mathrm{MHz}^{\mathrm{CDCl}} \mathrm{CDMS}_{3}, \mathrm{TM}\right) \delta: 3.5\left(\mathrm{~s}, 9 \mathrm{H}, \mathrm{OCH}_{3}\right)$, $5.1(\mathrm{~d}, 1 \mathrm{H}, \mathrm{CH}), 5.4(\mathrm{~d}, 1 \mathrm{H}, \mathrm{CH}), 6.1(\mathrm{~s}, 2 \mathrm{H}, \mathrm{Ar}-\mathrm{H}), 6.7-7.5(\mathrm{~m}$, $7 \mathrm{H}, \mathrm{Ar}-\mathrm{H}) .{ }^{13} \mathrm{C}-\mathrm{NMR}\left(300 \mathrm{MHz}, \mathrm{CDCl}_{3}\right) \delta: 162.2,150.3,141.2$, 137.2, 133.2, 127.4, 126.5, 125.3, 124.2, 121.4, 118.1, 108.2, 104, $63,62.2,56$.

3-chloro-4-(3,4-dimethoxyphenyl)-1-(naphthalen-3-yl)azetidin2-one $4 \mathrm{c}$

Molecular formula $\mathrm{C}_{21} \mathrm{H}_{18} \mathrm{ClNO}_{3}$; melting point $128-130{ }^{\circ} \mathrm{C}$; yield $60 \%$; IR $\left(\mathrm{KBr}, \mathrm{cm}^{-1}\right): 3,228.0(\mathrm{~N}-\mathrm{H}), 3,045.0(\mathrm{C}-\mathrm{H}), 1,749.4$ $(\mathrm{C}=\mathrm{O}), 1,672.2(\mathrm{CO}-\mathrm{NH}), 1,420.0(\mathrm{C}-\mathrm{N}), 832.8$ and $779.8(\mathrm{C}-\mathrm{C})$, $689.2(\mathrm{C}-\mathrm{Cl}) .{ }^{1} \mathrm{H}-\mathrm{NMR}\left(300 \mathrm{MHz}, \mathrm{CDCl}_{3}, \mathrm{TMS}=0\right) \delta: 3.6(\mathrm{~s}$, $\left.6 \mathrm{H}, \mathrm{OCH}_{3}\right), 5.2(\mathrm{~d}, 1 \mathrm{H}, \mathrm{CH}), 5.3(\mathrm{~d}, 1 \mathrm{H}, \mathrm{CH}), 6.6-7.6(\mathrm{~m}, 10 \mathrm{H}$, $\mathrm{Ar}-\mathrm{H})$.

3-chloro-4-(4-hydroxy-3-methoxyphenyl)-1-(naphthalen-3-yl) azetidin-2-one $4 \mathrm{~d}^{22}$

Molecular formula $\mathrm{C}_{20} \mathrm{H}_{16} \mathrm{ClNO}_{3}$; melting point $134-135^{\circ} \mathrm{C}$; yield $60 \% .{ }^{1} \mathrm{H}-\mathrm{NMR}\left(300 \mathrm{MHz}, \mathrm{CDCl}_{3}, \mathrm{TMS}=0\right) \delta: 3.7\left(\mathrm{~s}, 3 \mathrm{H}, \mathrm{OCH}_{3}\right)$, $4.9(\mathrm{~s}, 1 \mathrm{H}, \mathrm{OH}), 5.1(\mathrm{~d}, 1 \mathrm{H}, \mathrm{CH}), 5.4(\mathrm{~d}, 1 \mathrm{H}, \mathrm{CH}), 6.6-7.4(\mathrm{~m}$, $10 \mathrm{H}, \mathrm{Ar}-\mathrm{H}) .{ }^{13} \mathrm{C}-\mathrm{NMR}\left(300 \mathrm{MHz}, \mathrm{CDCl}_{3}\right) \delta: 162.1,151.2,143.2$, $141.4,137.2,133.2,127.4,126.5,125.3,124.3,121.4,120.6$, $118.1,116,112.2,108.2,63,62.2,56.2$.

3-chloro-1-(naphthalen-3-yl)-4-phenylazetidin-2-one 4e

Molecular formula $\mathrm{C}_{19} \mathrm{H}_{14} \mathrm{ClNO}$; melting point $118-119^{\circ} \mathrm{C}$; yield
60\%. IR ( $\left.\mathrm{KBr}, \mathrm{cm}^{-1}\right):$ 3,469.6 (NH), 3,054.0 (CH), 1,756.8 $(\mathrm{C}=\mathrm{O})$, 1,670.8 (CO-NH), 1,444.4 (C-N), 854.5, 679.2(C=C), $689.2(\mathrm{C}-$ Cl). ${ }^{1} \mathrm{H}-\mathrm{NMR}\left(300 \mathrm{MHz}, \mathrm{CDCl}_{3}, \mathrm{TMS}=0\right) \delta: 5.1(\mathrm{~d}, 1 \mathrm{H}, \mathrm{CH}), 5.4$ (d, 1H, CH), 6.8-7.4 (m, 12H, Ar-H).

3-chloro-1-(naphthalen-3-yl)-4-(3-nitrophenyl)azetidin-2-one 4f

Molecular formula $\mathrm{C}_{19} \mathrm{H}_{13} \mathrm{ClN}_{2} \mathrm{O}_{3}$; melting point $122-124{ }^{\circ} \mathrm{C}$; yield $70 \%$. IR $\left(\mathrm{KBr}, \mathrm{cm}^{-1}\right)$ : 3,449.5 (NH), 3,045.0 (CH); 1,757.1 $(\mathrm{C}=\mathrm{O}), 1,622.9(\mathrm{CO}-\mathrm{NH}), 1,446.7(\mathrm{CN}), 894.5,750.5$ and 677.8 $(\mathrm{C}=\mathrm{C}), 677.8(\mathrm{C}-\mathrm{Cl}) .{ }^{1} \mathrm{H}-\mathrm{NMR}\left(300 \mathrm{MHz}, \mathrm{CDCl}_{3}, \mathrm{TMS}=0\right) \delta$ : $5.4(\mathrm{~d}, 1 \mathrm{H}, \mathrm{CH}), 5.2$ (d, 1H, CH), 6.8-7.5 (m, 9H, Ar-H), 8.0 8.1( $\mathrm{m}, 2 \mathrm{H}, \mathrm{Ar}-\mathrm{H})$.

3-chloro-4-(4-chlorophenyl)-1-(naphthalen-3-yl)azetidin-2-one 4g

Molecular formula $\mathrm{C}_{19} \mathrm{H}_{13} \mathrm{Cl}_{2} \mathrm{NO}$; melting point $128-129^{\circ} \mathrm{C}$; yield 65\%; IR (KBr, cm $\left.{ }^{-1}\right): 3,238.0(\mathrm{~N}-\mathrm{H}), 3,049.0(\mathrm{C}-\mathrm{H}), 1,750.4$ $(\mathrm{C}=\mathrm{O}), 1,673.2(\mathrm{CO}-\mathrm{NH}), 1,422.0(\mathrm{C}-\mathrm{N}), 834.5$ and $779.8(\mathrm{C}-\mathrm{C})$, $619.9(\mathrm{C}-\mathrm{Cl}) .{ }^{1} \mathrm{H}-\mathrm{NMR}\left(300 \mathrm{MHz}, \mathrm{CDCl}_{3}, \mathrm{TMS}=0\right) \delta: 5.4(\mathrm{~d}$, $1 \mathrm{H}, \mathrm{CH}), 5.1(\mathrm{~d}, 1 \mathrm{H}, \mathrm{CH}), 6.8-7.4(\mathrm{~m}, 11 \mathrm{H}, \mathrm{Ar}-\mathrm{H})$.

4-(4-hydroxyphenyl)-3-(naphthalen-3-yl)thiazolidin-2-one 5a

Molecular formula $\mathrm{C}_{19} \mathrm{H}_{15} \mathrm{NO}_{2} \mathrm{~S}$; melting point $126-127^{\circ} \mathrm{C}$; yield $60 \% .{ }^{1} \mathrm{H}-\mathrm{NMR}\left(300 \mathrm{MHz}, \mathrm{CDCl}_{3}, \mathrm{TMS}=0\right) \delta: 5.0(\mathrm{~s}, 1 \mathrm{H}, \mathrm{OH})$, $4.8(\mathrm{t}, 1 \mathrm{H}, \mathrm{CH}), 3.25\left(\mathrm{dd}, 2 \mathrm{H}, \mathrm{J}=5.6 \mathrm{~Hz}, \mathrm{CH}_{2}\right), 6.8-7.4(\mathrm{~m}, 11 \mathrm{H}$, $\mathrm{Ar}-\mathrm{H}) .{ }^{13} \mathrm{C}-\mathrm{NMR}\left(300 \mathrm{MHz}, \mathrm{CDCl}_{3}\right) \delta: 170.1,156.4,141.2$, $136.4,133.2,128,127.4,126.1,125.4,124,121.4,118.2,115$, $108.6,55,35.2$.

4-(3,4,5-trimethoxyphenyl)-3-(naphthalen-3-yl)thiazolidin-2-one $5 b$

Molecular formula $\mathrm{C}_{22} \mathrm{H}_{21} \mathrm{NO}_{4} \mathrm{~S}$; melting point $128-130{ }^{\circ} \mathrm{C}$; yield $65 \%$. ${ }^{1} \mathrm{H}-\mathrm{NMR}\left(300 \mathrm{MHz}, \mathrm{CDCl}_{3}, \mathrm{TMS}=0\right) \delta: 3.25(\mathrm{dd}, 2 \mathrm{H}, \mathrm{J}=$ $\left.5.6 \mathrm{~Hz}, \mathrm{CH}_{2}\right), 3.7\left(\mathrm{~s}, 9 \mathrm{H}, \mathrm{OCH}_{3}\right), 5.2(\mathrm{t}, 1 \mathrm{H}, \mathrm{CH}), 6.2-7.4(\mathrm{~m}, 9 \mathrm{H}$, $\mathrm{Ar}-\mathrm{H})$.

4-(3,4-dimethoxyphenyl)-3-(naphthalen-3-yl)thiazolidin-2-one 5c

Molecular formula $\mathrm{C}_{21} \mathrm{H}_{19} \mathrm{NO}_{3} \mathrm{~S}$; melting point $133-134{ }^{\circ} \mathrm{C}$; yield $58 \% .{ }^{1} \mathrm{H}-\mathrm{NMR}\left(300 \mathrm{MHz}, \mathrm{CDCl}_{3}, \mathrm{TMS}=0\right) \delta: 3.27(\mathrm{dd}, 2 \mathrm{H}, \mathrm{J}$ $\left.=5.7 \mathrm{~Hz}, \mathrm{CH}_{2}\right), 3.7\left(\mathrm{~s}, 6 \mathrm{H}, \mathrm{OCH}_{3}\right), 5.2(\mathrm{t}, 1 \mathrm{H}, \mathrm{CH}), 6.6-7.4(\mathrm{~m}$, $10 \mathrm{H}, \mathrm{Ar}-\mathrm{H})$.

4-(4-hydroxy-3-methoxyphenyl)-3-(naphthalen-3-yl)thiazolidin2-one $5 \mathrm{~d}^{22}$

Molecular formula $\mathrm{C}_{20} \mathrm{H}_{17} \mathrm{NO}_{3} \mathrm{~S}$; melting point $137-138^{\circ} \mathrm{C}$; yield $55 \% .{ }^{1} \mathrm{H}-\mathrm{NMR}\left(300 \mathrm{MHz}, \mathrm{CDCl}_{3}, \mathrm{TMS}=0\right) \delta: 3.32(\mathrm{dd}, 2 \mathrm{H}, \mathrm{J}=$ $\left.5.6 \mathrm{~Hz}, \mathrm{CH}_{2}\right), 3.6\left(\mathrm{~s}, 3 \mathrm{H}, \mathrm{OCH}_{3}\right), 4.9(\mathrm{~s}, 1 \mathrm{H}, \mathrm{OH}), 5.1(\mathrm{t}, 1 \mathrm{H}, \mathrm{CH})$, 6.6-7.4 (m, 10H, Ar-H).

3-(naphthalen-3-yl)-4-phenylthiazolidin-2-one 5e

Molecular formula $\mathrm{C}_{19} \mathrm{H}_{15} \mathrm{NOS}$; melting point $134-135{ }^{\circ} \mathrm{C}$; yield 
Table 1. Molecular properties of the $3 \mathrm{a}-\mathrm{g}, 4 \mathrm{a}-\mathrm{g}$ and $5 \mathrm{a}-\mathrm{g}$ compounds

\begin{tabular}{|c|c|c|c|c|c|c|}
\hline Compound & $\mathrm{Ar}$ & LogP & TPSA & Natom & nON & nOHNH \\
\hline $3 a$ & $-4 \mathrm{OHC}_{6} \mathrm{H}_{4}$ & 4.17 & 32.59 & 19 & 2 & 1 \\
\hline $3 b$ & $-3,4,5$-triOCH ${ }_{3} \mathrm{C}_{6} \mathrm{H}_{2}$ & 4.29 & 40.07 & 24 & 4 & 0 \\
\hline $3 c$ & $-3,4-\operatorname{diOCH}_{3} \mathrm{C}_{6} \mathrm{H}_{3}$ & 4.30 & 30.83 & 22 & 3 & 0 \\
\hline $3 d$ & $-4 \mathrm{OH}, 3-\mathrm{OCH}_{3} \mathrm{C}_{6} \mathrm{H}_{3}$ & 3.99 & 41.83 & 21 & 3 & 1 \\
\hline $3 e$ & $-\mathrm{C}_{6} \mathrm{H}_{5}$ & 4.65 & 12.36 & 18 & 1 & 0 \\
\hline $3 f$ & $-3 \mathrm{NO}_{2} \mathrm{C}_{6} \mathrm{H}_{4}$ & 4.59 & 58.19 & 21 & 4 & 0 \\
\hline $3 g$ & $-4 \mathrm{ClC}_{6} \mathrm{H}_{4}$ & 5.33 & 12.36 & 19 & 1 & 0 \\
\hline $4 a$ & $-4 \mathrm{OHC}_{6} \mathrm{H}_{4}$ & 4.10 & 40.54 & 23 & 3 & 1 \\
\hline $4 b$ & $-3,4,5-$ triOCH ${ }_{3} \mathrm{C}_{6} \mathrm{H}_{2}$ & 4.21 & 48.01 & 28 & 5 & 0 \\
\hline $4 c$ & $-3,4-\mathrm{diOCH}_{3} \mathrm{C}_{6} \mathrm{H}_{3}$ & 4.23 & 38.78 & 26 & 4 & 0 \\
\hline $4 d$ & $-4 \mathrm{OH}, 3-\mathrm{OCH}_{3} \mathrm{C}_{6} \mathrm{H}_{3}$ & 3.92 & 49.77 & 25 & 4 & 1 \\
\hline $4 \mathrm{e}$ & $-\mathrm{C}_{6} \mathrm{H}_{5}$ & 4.58 & 20.31 & 22 & 2 & 0 \\
\hline $4 f$ & $-3 \mathrm{NO}_{2} \mathrm{C}_{6} \mathrm{H}_{4}$ & 4.51 & 66.13 & 25 & 5 & 0 \\
\hline $4 g$ & $-4 \mathrm{ClC}_{6} \mathrm{H}_{4}$ & 5.26 & 20.31 & 23 & 2 & 0 \\
\hline $5 a$ & $-4 \mathrm{OHC}_{6} \mathrm{H}_{4}$ & 4.58 & 40.54 & 23 & 3 & 1 \\
\hline $5 b$ & $-3,4,5$-triOCH ${ }_{3} \mathrm{C}_{6} \mathrm{H}_{2}$ & 4.69 & 48.01 & 28 & 5 & 0 \\
\hline $5 c$ & $-3,4-\mathrm{diOCH}_{3} \mathrm{C}_{6} \mathrm{H}_{3}$ & 4.70 & 38.78 & 26 & 4 & 0 \\
\hline $5 d$ & $-4 \mathrm{OH}, 3-\mathrm{OCH}_{3} \mathrm{C}_{6} \mathrm{H}_{3}$ & 4.40 & 49.77 & 25 & 4 & 1 \\
\hline $5 e$ & $-\mathrm{C}_{6} \mathrm{H}_{5}$ & 5.06 & 20.31 & 22 & 2 & 0 \\
\hline $5 f$ & $-3 \mathrm{NO}_{2} \mathrm{C}_{6} \mathrm{H}_{4}$ & 4.99 & 66.13 & 25 & 5 & 0 \\
\hline $5 g$ & $-4 \mathrm{ClC}_{6} \mathrm{H}_{4}$ & 5.74 & 20.31 & 23 & 2 & 0 \\
\hline
\end{tabular}

$68 \% .{ }^{1} \mathrm{H}-\mathrm{NMR}\left(300 \mathrm{MHz}, \mathrm{CDCl}_{3}, \mathrm{TMS}=0\right) \delta: 3.34(\mathrm{dd}, 2 \mathrm{H}, \mathrm{J}=$ $\left.5.8 \mathrm{~Hz}, \mathrm{CH}_{2}\right), 5.1(\mathrm{t}, 1 \mathrm{H}, \mathrm{CH}), 6.8-7.5(\mathrm{~m}, 12 \mathrm{H}, \mathrm{Ar}-\mathrm{H})$.

3-(naphthalen-3-yl)-4-(3-nitrophenyl)thiazolidin-2-one 5f

Molecular formula $\mathrm{C}_{19} \mathrm{H}_{14} \mathrm{~N}_{2} \mathrm{O}_{3} \mathrm{~S}$; melting point $121-122{ }^{\circ} \mathrm{C}$; yield $60 \%$. ${ }^{1} \mathrm{H}-\mathrm{NMR}\left(300 \mathrm{MHz}, \mathrm{CDCl}_{3}, \mathrm{TMS}=0\right) \delta: 3.35(\mathrm{dd}, 2 \mathrm{H}, \mathrm{J}=$ $\left.5.74 \mathrm{~Hz}, \mathrm{CH}_{2}\right), 5.2(\mathrm{t}, 1 \mathrm{H}, \mathrm{CH}), 6.8-7.6(\mathrm{~m}, 9 \mathrm{H}, \mathrm{Ar}-\mathrm{H}), 8.1-8.2$ (m, 2H, Ar-H).

\section{4-(4-chlorophenyl)-3-(naphthalen-3-yl)thiazolidin-2-one 5g}

Molecular formula $\mathrm{C}_{19} \mathrm{H}_{14} \mathrm{ClNOS}$; melting point $136-137{ }^{\circ} \mathrm{C}$; yield $70 \%$. ${ }^{1} \mathrm{H}-\mathrm{NMR}\left(300 \mathrm{MHz}, \mathrm{CDCl}_{3}\right.$, TMS $\left.=0\right) \delta: 3.36(\mathrm{dd}$, $\left.2 \mathrm{H}, \mathrm{J}=5.75, \mathrm{CH}_{2}\right), 5.0(\mathrm{t}, 1 \mathrm{H}, \mathrm{CH}), 6.8-7.6(\mathrm{~m}, 11 \mathrm{H}, \mathrm{Ar}-\mathrm{H})$.

\section{In vitro antimicrobial activity test}

The in vitro antimicrobial potential of all newly prepared analogs was tested at Guru Gobind Singh College of Pharmacy, (Yamuna Nagar, India) using the Agar plate diffusion antimicrobial bioassay. Antimicrobial activity of all the synthesized analogs (3a-g, 4a-g and $5 \mathrm{a}-\mathrm{g}$ ) were tested against four standard bacterial cultures (i.e. Bacillus subtilis MTCC 121, Staphylococcus aureus MTCC 96, Escherichia coli MTCC 739 and Pseudomonas aeruginosa MTCC 2453) and one fungal strain (Candida albicans MTCC 3017) ac- cording to the literature as described. ${ }^{24}$ The stock solutions for all new prepared compounds $(3 \mathrm{a}-\mathrm{g}, 4 \mathrm{a}-\mathrm{g}$ and $5 \mathrm{a}-\mathrm{g} ; 5,000 \mu \mathrm{g} / \mathrm{mL}$ each) were prepared in DMSO $(50 \mu \mathrm{g}$ per well, i.e. $250 \mu \mathrm{g})$ and the activity was determined by measuring the zone of inhibition. The strength (i.e. $50 \mu \mathrm{g} / \mathrm{mL}$ ) was used as positive control for the bioassay. The antibacterial and antifungal potentials of the prepared analogs were compared with the standard drugs ampicillin $(200 \mu \mathrm{g} /$ $\mathrm{mL})$ and amphotericin B $(500 \mu \mathrm{g} / \mathrm{mL})$, respectively. Activity was determined by measuring the diameter of zones showing complete inhibition (mm). Growth inhibition was calculated with reference to the positive control.

\section{Results and discussion}

\section{Chemistry}

All of the new azetidinone and thiazolidione analogs of napthylamine were synthesized successfully, with the subsequent aim of finding new compounds with promising antibacterial activities. The synthetic methodology is shown in Scheme 1. The purity and structures of all the synthesized compounds were elucidated on the basis of their spectral data (i.e. IR, ${ }^{1} \mathrm{H}-\mathrm{NMR}$ and $\left.{ }^{13} \mathrm{C}-\mathrm{NMR}\right)$. The IR spectra of $3 \mathrm{a}-\mathrm{g}$ compounds displayed the characteristic peaks in the region of $1,440-1,514 \mathrm{~cm}^{-1}$ indicating the formation of a Schiff base $(-\mathrm{H}-\mathrm{C}=\mathrm{N})$. The appearance of peaks in the region of 1,550 $1,687 \mathrm{~cm}^{-1}$ confirmed the presence of $\mathrm{C}=\mathrm{O}$ in $4 \mathrm{a}-\mathrm{g}$ and $5 \mathrm{a}-\mathrm{g}$. The structural assignments were further supported by their ${ }^{1} \mathrm{H}-\mathrm{NMR}$ 
Table 2. Antibacterial and antifungal potential of all the synthesized analogs

\begin{tabular}{|c|c|c|c|c|c|c|c|c|c|c|}
\hline \multirow[t]{2}{*}{ Compound } & \multicolumn{2}{|c|}{$\begin{array}{l}\text { B. subtilis } \\
\text { MTCC } 121\end{array}$} & \multicolumn{2}{|c|}{$\begin{array}{l}\text { S. aureus } \\
\text { MTCC } 96\end{array}$} & \multicolumn{2}{|c|}{$\begin{array}{c}\text { E. coli } \\
\text { MTCC } 739\end{array}$} & \multicolumn{2}{|c|}{$\begin{array}{l}\text { P. aeruginosa } \\
\text { MTCC } 2453\end{array}$} & \multicolumn{2}{|c|}{$\begin{array}{l}\text { C. albicans } \\
\text { MTCC } 3017\end{array}$} \\
\hline & $24 h$ & $48 h$ & $24 h$ & $48 h$ & $24 h$ & 48h & $24 h$ & 48h & $24 h$ & $48 \mathrm{~h}$ \\
\hline $3 a$ & $10 \mathrm{~mm}$ & - & - & - & - & - & - & - & - & - \\
\hline $3 b$ & - & - & - & - & - & - & - & - & $7 \mathrm{~mm}$ & - \\
\hline $3 c$ & - & - & - & - & - & - & - & - & - & - \\
\hline $3 d$ & - & - & - & - & - & - & - & - & - & - \\
\hline $3 e$ & $12 \mathrm{~mm}$ & - & - & - & - & - & - & - & - & - \\
\hline $3 f$ & - & - & - & - & - & - & - & - & - & - \\
\hline $3 g$ & - & - & - & - & - & - & - & - & - & - \\
\hline $4 a$ & $9 \mathrm{~mm}$ & $9 \mathrm{~mm}$ & $12 \mathrm{~mm}$ & $10 \mathrm{~mm}$ & - & - & $10 \mathrm{~mm}$ & $10 \mathrm{~mm}$ & - & - \\
\hline $4 b$ & - & - & - & - & - & - & - & - & - & - \\
\hline $4 c$ & - & - & - & - & - & - & - & - & - & - \\
\hline $4 d$ & $12 \mathrm{~mm}$ & - & $15 \mathrm{~mm}$ & - & - & - & - & - & - & - \\
\hline $4 e$ & $10 \mathrm{~mm}$ & $10 \mathrm{~mm}$ & - & - & $19 \mathrm{~mm}$ & - & $9 \mathrm{~mm}$ & - & - & - \\
\hline $4 f$ & $16 \mathrm{~mm}$ & $15 \mathrm{~mm}$ & $15 \mathrm{~mm}$ & $15 \mathrm{~mm}$ & - & - & $19 \mathrm{~mm}$ & - & $13 \mathrm{~mm}$ & - \\
\hline $4 g$ & $12 \mathrm{~mm}$ & $15 \mathrm{~mm}$ & - & - & - & - & $14 \mathrm{~mm}$ & - & - & - \\
\hline $5 a$ & - & - & $12 \mathrm{~mm}$ & - & - & - & - & - & - & - \\
\hline $5 b$ & - & - & - & - & - & - & - & - & $9 \mathrm{~mm}$ & - \\
\hline $5 c$ & - & - & - & - & - & - & - & - & - & - \\
\hline $5 d$ & - & - & - & - & $20 \mathrm{~mm}$ & $20 \mathrm{~mm}$ & - & - & - & - \\
\hline $5 e$ & $13 \mathrm{~mm}$ & - & - & - & - & - & - & - & $13 \mathrm{~mm}$ & - \\
\hline $5 f$ & - & - & - & - & - & - & - & - & - & - \\
\hline $5 g$ & $13 \mathrm{~mm}$ & - & - & - & $11 \mathrm{~mm}$ & - & - & - & - & - \\
\hline \multirow[t]{2}{*}{ Positive control } & $27 \mathrm{~mm}$ & $22 \mathrm{~mm}$ & $44 \mathrm{~mm}$ & $45 \mathrm{~mm}$ & $15 \mathrm{~mm}$ & $17 \mathrm{~mm}$ & - & - & $16 \mathrm{~mm}$ & $15 \mathrm{~mm}$ \\
\hline & \multicolumn{2}{|c|}{ 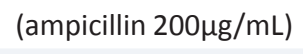 } & \multicolumn{2}{|c|}{ (ampicillin $200 \mu \mathrm{g} / \mathrm{mL}$ ) } & \multicolumn{3}{|c|}{ (ampicillin $200 \mu \mathrm{g} / \mathrm{mL}$ ) } & & \multicolumn{2}{|c|}{ (amphotericin B, 500 $\mathrm{gg} / \mathrm{mL}$ ) } \\
\hline DMSO (control) & - & - & - & - & - & - & - & - & - & - \\
\hline
\end{tabular}

spectra, and all the synthesized compounds were found to be in conformity with the structures envisaged. The molecular properties of the title compounds, such as log P, topological polar surface area (TPSA), number of atoms other than hydrogen (Natom), number of hydrogen bond donor (nON) and number of $\mathrm{H}-$ bond acceptor (n OHNH), were calculated by the online Molinspiration software and the data recorded are given in Table $1 .{ }^{25}$

\section{Antibacterial activity}

The antibacterial potential of prepared analogs was compared with the standard drug ampicillin, whereas the antifungal potential was compared with the standard drug amphotericin B (Table 2). The observation was made over 2 days and the "-" sign signifies no zone of inhibition (ZOI). In many cases, on the first day, ZOI was observed but had disappeared on the next day.

Schiff base analogs in this series $(3 \mathrm{a}-\mathrm{g})$ showed no activity against any of the bacteria tested. Three compounds, $4 \mathrm{a}, 4 \mathrm{e}$ and $4 \mathrm{~g}$, were found to exhibit broad spectrum activity. Compound $4 \mathrm{~d}$ showed good activity against $B$. subtilis MTCC $121(12 \mathrm{~mm})$ and moderate activity against S.aureus MTCC 96 (15 mm). However, compound $4 \mathrm{f}$ showed a wide spectrum of antimicrobial activity, but it may be cytotoxic. Compounds $3 \mathrm{~b}, 5 \mathrm{~b}$ and $5 \mathrm{e}$ were found to be active against $C$. albicans MTCC 3017, with ZOIs at $24 \mathrm{~h} 7$ $\mathrm{mm}, 9 \mathrm{~mm}$ and $13 \mathrm{~mm}$, respectively. Although ampicillin is a broad spectrum antibiotic, it is inactive against Pseudomonas species at the tested concentration, which may be due to some mutation.

\section{Future research directions}

$\mathrm{N}$-heterocyclic nucleus and its analogues have attracted the interest of many medicinal chemists, and because of this many modifications have been carried out in last decade. Keeping this in view, the work in this study was carried out with different approaches to develop better analogs of napthylamine analogs having azetidin2-one and thiazolidin-4-one moiety with comparable antimicrobial activity. The promising activity of these compounds, along with the other activity data obtained during the study, can also be useful in establishing the structure activity relationships and for the development of newer and more potent antimicrobial compounds 
J Explor Res Pharmacol

which will able to act against multidrug resistance.

\section{Conclusions}

In summary, the present investigation describes the synthesis and biological evaluation of naphthylamine analogs having azetidin2-one and thiazolidin-4-one heterocyclic nucleus/moiety. All the prepared analogs were characterized by suitable methods, such as IR and ${ }^{1} \mathrm{H}-\mathrm{NMR}$. All spectral data were in accordance with assumed structures. The three compounds $4 \mathrm{a}, 4 \mathrm{e}, 4 \mathrm{~g}$ and $4 \mathrm{f}$ exhibited broad spectrum activity.

\section{Acknowledgments}

The authors extend their heartfelt gratitude to IK Gujral Punjab Technical University, Kapurthala, Punjab, India and Dr. Jagdeep Singh Dua, Shivalik College of Pharmacy, Nangal, Punjab, India for valuable suggestions and moral support.

\section{Conflict of interest}

The authors have no conflict of interests related to this publication.

\section{Author contributions}

Overall experimental work (BC), preparation of manuscript (BC), Analysis (AK), revision of analysis (RPK), research guidance (DNP).

\section{References}

[1] Rokade YB, Sayyed RZ. Naphthalene derivatives: a new range of antimicrobials with high therapeutic value. RasayanJ Chem 2009;2(4):972-980.

[2] Delgado JN. Wilson and Gisvold's textbook of organic medicinal and pharmaceutical chemistry. Lippincott: Williams and Wilkins, Philadelphia. 2004;255-257.

[3] Dhar DN, Taploo CL. Schiff bases and their applications. J Scilnd Res 1982;1(8):501-506.

[4] Bringmann G, Dreyer M, Faber JH, Dalsgaard PW, Staerk D, Jaroszewski JW, et al. Ancistrotanzanine $C$ and related 5,1'- and 7,3'-coupled naphthylisoquinoline alkaloids from Ancistrocladus tanzaniensis. J Nat Prod 2004;67(5):743-748. doi:10.1021/np0340549.

[5] de Souza AO, Galett FCS, Silva CL, Bicalho B, Parma MM, Fonseca SF, et al. Antimycobacterial and cytotoxicity activity of synthetic and natural compounds. Quim Nova 2007;30(7):1563-1566. doi:10.1590/ S0100-40422007000700012.

[6] da Silva CM, da Silva DL, Modolo LV, Alves RB, de Resende MA, Martins CVB, et al. Schiff bases: A short review of their antimicrobial activities. J Adv Res 2011;2(1):1-8. doi:10.1016/j.jare.2010.05.004.

[7] Lin SJ, Tsai WJ, Chiou WF, Yanga TH, Yanga LM. Selective COX-2 inhibitors. Part 2: Synthesis and biological evaluation of 4-benzylideneamino- and 4-phenyliminomethyl-benzenesulfonamides. Bioorg Med Chem 2008;16(5):2697-2706. doi:10.1016/j.bmc.2007.11.033.

[8] Gemma S, Savini L, Altarelli M, Tripaldi P, Chiasserini L, Coccone SS,
Chopra B. et al: Napthylamine analogs having heterocyclic moiety

et al. Development of antitubercular compounds based on a 4-quinolylhydrazone scaffold. Further structure-activity relationship studies. Bioorg Med Chem 2009;17(16):6063-6072. doi:10.1016/j. bmc.2009.06.051.

[9] Hearn MJ, Cynamon MH, Chen MF, Coppins R, Davis J, Kang HJ, et al. Preparation and antitubercular activities in vitro and in vivo of nove Schiff bases of isoniazid. Eur J Med Chem 2009;44(10):4169-4178. doi:10.1016/j.ejmech.2009.05.009.

[10] Raparti V, Chitre T, Bothara K, Kumar V, Dangre S, Khachane C, et al. Novel 4-(morpholin-4-yl)-N'-(arylidene)benzohydrazides: Synthesis, antimycobacterial activity and QSAR investigations. Eur J Med Chem 2009;44(10):3954-3960. doi:10.1016/j.ejmech.2009.04.023.

[11] Cheng LX, Tang JJ, Luo H, Jin XL, Dai F, Yang J, et al. Antioxidant and antiproliferative activities of hydroxyl-substituted Schiff bases. Bioorg Med Chem Lett 2010;20(8):2417-2420. doi:10.1016/j. bmcl.2010.03.039.

[12] Jarrahpour A, Khalili D, Clercq ED, Salmi C, Brunel JM. Synthesis, Antibacterial, Antifungal and Antiviral Activity Evaluation of Some New bis-Schiff Bases of Isatin and Their Derivatives. Molecule 2007;12(8):1720-1730. doi:10.3390/12081720.

[13] Khan KM, Ambreen N, Hussain S, Perveen S, Choudhary MI. Schiff bases of 3-formylchromone as thymidine phosphorylase inhibitors. Bioorg Med Chem 2009;17(8):2983-2988. doi:10.1016/j. bmc.2009.03.020.

[14] Tarafder MT, Kasbollah A, Saravanan N, Crouse KA, Ali AM, Tin Oo K. S-methyldithiocarbazate and its Schiff bases: evaluation of bondings and biological properties. Biochem Mol Biol Biophys 2002;6:85-91. doi:10.1080/10258140290027207

[15] Kucukguzel I, Kucukguzel SG, Rollas S, Sanis GO, Ozdemir O, Bayrak I, et al. Synthesis of some 3-(Arylalkylthio)-4-alkyl/aryl5-(4-aminophenyl)-4H-1,2,4-triazole derivatives and their anticonvulsant activity. Farmaco 2004;59:893-901. doi:10.1016/j.farmac.2004.07.005.

[16] Vicini P, Geronikaki A, Incerti M, Busonera B, Poni G, Kabras CA, et al. Synthesis and Biological Evaluation of Benzo[d]isothiazole, Benzothiazole and Thiazole Schiff Bases. Bioorg Med Chem 2003;11(22):47854789. doi:10.1016/S0968-0896(03)00493-0.

[17] Bekircan O, Kahveci B, Kucuk M. Synthesis and anticancer evaluation of some new unsymmetrical 3, 5-diaryl-4H-1, 2, 4-triazole derivatives. Tur J Chem 2006;30:29-40.

[18] Guo Z, Xing R, Liu S, Zhong Z, Ji X, Wang L, et al. Antifungal properties of Schiff bases of chitosan, $\mathrm{N}$-substituted chitosan and quaternized chitosan. Carbohydr Res 2007;342(10):1329-1332. doi:10.1016/j. carres.2007.04.006.

[19] Jain AK, Vaidya A, Ravichandran V, Kashaw SK, Agrawal RK. Recent developments and biological activities of thiazolidinone derivatives: a review. Bioorg Med Chem 2012;20(11):3378-95. doi:10.1016/j. bmc.2012.03.069.

[20] Zachariah SM, Ramkumar M, George N, Ashif MS. Azetidinones: An overview. Int J Pharm Sci Rev Res 2015;30(1):211-218.

[21] De Rosa M, Vigliotta G, Palma G, Saturnino C, Soriente A. Novel penicillin-type analogues bearing a variable substituted 2-Azetidinone ring at position 6: synthesis and biological evaluation. Molecules 2015;20(12):22044-22057. doi:10.3390/molecules201219828.

[22] Sharma S, Singh T, Mittal R, Saxena KK, Srivastava VK, Kumar A. A study of anti-inflammatory activity of some novel $\alpha$-Amino naphthalene and $\beta$-Amino naphthalene derivatives. Arch Pharm (Weinheim) 2006;339(3):145-152. doi:10.1002/ardp.200500215.

[23] Baig MZK, Pallikonda G, Trivedi P, Tulichala RNP, Ghosh B, Chakravarty M. Metal-free arylation to access distinct anthracenylphosphonates and anticancer activities for these and allied phosphonates. Chem 2016;1(14):4332-4339. doi:10.1002/slct.201600978.

[24] Atta-ur-Rahman MI, Choudhary WJT. Bioassay Techniques for Drug Development. The Netherlands: Harwood Academic Publishers. 2001;15-22.

[25] http://www.molinspiration.com 